\title{
Evolution des inégalités devant la mort (1992-2012) selon les caractéristiques de l'enfant: cas des enfants de moins de 5 ans au Niger.
}

\author{
Zourkaleini Younoussi \\ Université de Zinder, Niger \\ younoussi@gmx.com
}

\begin{abstract}
Resume
L'examen des différents résultats des Enquêtes Démographiques et de Santé (EDS) réalisées au Niger permet de constater que le niveau de la mortalité infanto-juvénile a diminué régulièrement au cours des vingt dernières années en passant de $318 \%$ à $127 \%$ entre 1992 et 2012 . On note une mortalité différentielle des enfants de moins de cinq ans selon l'âge, le sexe, le poids à la naissance, et l'intervalle inter-génésique de l'enfant. A partir des résultats des quatre EDS (1992, 1998, 2006, 20I2) et sur la base de calculs d'indice d'évolution notre objectif est de mettre en évidence les évolutions des inégalités selon ces différentes variables en fonction des différents quotients de mortalité (néonatale, postnéonatale, infantile, juvénile). Nos résultats montrent que la baisse de la mortalité des enfants s'accompagne souvent d'une aggravation des inégalités devant la mort.
\end{abstract}

Mots clés: Mortalité, enfance, inégalités, Niger

\section{Abstract}

Evolution of the disparities in front of the death (1992-20/2) according to the characteristics of the child: case of the children of less than 5 years in Niger. The examination of the various results of the Demographic and Health surveys (DHS) realized in Niger allows noticing that the level of the young mortality decreased regularly during the last twenty years in passing from $318 \%$ o to $127 \%$ o between 1992 and 2012. We note a differential mortality of the less than five-year-old children according to the age, the sex, the weight at the birth, and the preceding birth interval. From the results of four DHS (on 1992, on 1998, on 2006, on 2012) and on the basis of calculations of indication of evolution our objective is to highlight the evolutions of the disparities according to these various variables according to the various quotients of mortality (neonatal, post-neonatal, infantile, young). Our results show that the reduction in the mortality of the children of often comes along with a worsening of the disparities in front of the death.

Keywords: Mortality, child, disparities, Niger

\section{Introduction}

Au Niger, pour assurer un suivi-évaluation des différentes politiques et programmes de santé mis en œuvre, en particulier dans le domaine de la santé, au cours de la période 1992-2012, des séries d'Enquêtes Démographiques et de Santé (EDS) de couverture nationale ont été réalisées. Les données collectées à partir de ces enquêtes permettent de reconstruire les tendances de la mortalité des jeunes enfants (la mortalité entre 0 et 5 ans). Ainsi, selon les résultats de ces enquêtes, le quotient de mortalité des enfants de moins de cinq ans est passé de 318 à 127 pour mille naissances vivantes, entre 1992 et 2012 . Pendant la même période, le quotient de mortalité infantile est passé de 123 à $5 \mathrm{I}$ pour mille alors que celui de la mortalité juvénile est passé de 223 à 81 pour mille (INS et ICF, 20I3). De façon générale, toutes les composantes de la mortalité des jeunes enfants, ont donc enregistré une baisse importante.

Cette baisse sensible de la mortalité des enfants classe le Niger parmi les rares pays de l'Afrique de l'Ouest qui ont atteint la cible du $4^{\text {iemme }}$ Objectif du Millénaire pour le Développement qui visait à réduire de 2/3 la mortalité des enfants de moins de cinq ans entre 1990 et 2015 (You et al., 2015). Cette rapide baisse de la mortalité des enfants de moins de cinq ans s'accompagne-t-elle d'une réduction des inégalités, selon les caractéristiques de l'enfant, devant la mort ? Ou alors, a-t-elle permis une augmentation des inégalités devant la mort selon les caractéristiques des enfants ?

L'objectif de cette étude est d'examiner, à partir des données des quatre EDS que le pays a connues, les évolutions des inégalités observées au niveau de 
certaines caractéristiques des enfants. II s'agit du sexe, du rang et du poids à la naissance de l'enfant mais aussi de l'intervalle qui précède la naissance de l'enfant. Le choix de ces variables se justifie par leur lien avec les actions sociales et les politiques publiques supposées être à la base de la relative baisse de la mortalité infantile, juvénile et infantojuvénile. Pour chacun de ces déterminants il s'agit de mettre en évidence, au cours de la période, l'atténuation et/ou l'augmentation des inégalités des enfants devant la mort.

Dans une première section, nous présentons une brève revue de la littérature et du cadre théorique de la mortalité des enfants. La deuxième section précise la méthodologie utilisée. La troisième section retrace l'évolution de la mortalité des enfants de moins de cinq ans au cours des vingt dernières années. La quatrième section est consacrée aux variations des inégalités devant la mort suivant les caractéristiques liées aux enfants retenues dans le cadre de cette étude.

\section{Revue de littérature et cadre théorique}

Les analyses explicatives de la diminution de la mortalité se sont peu à peu développées au sein d'un champ scientifique spécifique où se dégage une certaine pluralité des théories (Vallin, 1989 ; Tabutin, 1995 ; Kabir et al., 20II). D'après la synthèse développée par Vallin (1989), la théorie initiale et dominante jusqu'à la fin des années soixante met en avant le rôle majeur des technologies sanitaires (Razzel, 1965), en déniant toute importance, dans la diminution de la mortalité, à la croissance économique et à la progression des revenus individuels (Demeny, 1965 ; Stolnitz, 1965).

Une deuxième théorie s'est située dans une perspective rigoureusement inverse en affirmant que seule l'élévation du niveau de vie pouvait déterminer une diminution de la mortalité ; le modèle le plus achevé de cette théorie se trouvant développé par McKeown (1976) instituant les progrès dans l'alimentation comme source essentielle de l'augmentation de l'espérance de vie.

Le radicalisme de ces deux thèses est apparue dépassé dès les années 1980 (Chesnais, 1986), et l'articulation de plusieurs facteurs est devenue l'idée dominante et inspiratrice de nombreux travaux où se trouvent privilégiées certaines variables clés (revenu, alimentation, instruction, développement de la santé publique, transfert technologique, urbanisation) (Rutstein, 2000 ; Becher et al., 2004, Sartorius et Sartorius K. , 2014).

Concernant la mortalité des enfants, en particulier la mortalité infantile, les recherches trouvent un certain aboutissement dans le modèle conceptuel de Mosley (1985) qui met en avant à propos de la relation malnutrition-infection, considérée comme la principale cause médicale de décès infantile, le rôle fondamental : a) de deux variables indépendantes majeures : la qualité des pratiques individuelles et collectives d'une part, les moyens disponibles pour la mise en œuvre de ces pratiques d'autre part ; b) de cinq groupes de variables intermédiaires : les ressources alimentaires, la qualité de l'environnement, les traumatismes, la fécondité et la lutte contre les maladies.

Les caractéristiques liées à la mère (l'âge, la situation sociale, l'ethnie), et d'autres, plus liées aux conditions de l'accouchement (naissance prématurée, naissances multiples, assistance à l'accouchement ....) font partis des multiples facteurs de la mortalité infantile identifiés depuis longtemps et qui persistent encore(Croze, 1963 ; Dinh, 1998 ; Beck, 2009 ; Hamadou Daouda, 20I2 ; Esso Lasme, 2013 ; Brinda et al., 20I5).

Les facteurs culturels occupent également une place de choix dans l'étude des déterminants de la mortalité des enfants (Akoto, 1989 et 1993; Akoto et Tabutin, 1989 ; Cantrelle et Locoh, 1990 ; Gakidou et al., 2010 ; Hamadou Daouda, 2012 ; Lankoande et al. 2016). Les traditions, normes et pratiques culturelles agissent sur la mortalité des enfants à travers le comportement, l'attitude et les croyances qu'elles induisent auprès des individus. Les croyances étiologiques, par exemple, influencent beaucoup la nature du système de soins auquel on va recourir selon la cause attribuée à la maladie. La perception qu'ont les individus vis à vis de l'enfant agit sur les attitudes et comportements à son égard. Ainsi, un enfant né en dehors de l'union conjugale est soumis à un risque de décès supérieur ou égal à celui né dans le mariage.

Le comportement des individus est influencé également par leur niveau d'instruction qui a un impact sur la mortalité, des adultes comme des enfants (Gakidou et al., 20l0). Cette action se réalise par le canal de toute une série de variables telles la rupture avec certaines pratiques traditionnelles nuisibles à la santé, la facilité de s'adapter au monde "moderne", la sensibilisation aux problèmes d'hygiène et d'alimentation, la modification des soins familiaux à accorder aux enfants (Kabir et al., 201 I ; Maîga et Bocquier, 2016). Ainsi, au Niger, partout en Afrique comme ailleurs dans le monde, le niveau d'instruction des parents " explique " une part non négligeable de la variation de la mortalité des enfants et, de manière générale, il existe une relation négative entre cette variable et la mortalité des enfants. A une augmentation du niveau d'études correspond en général une baisse de la mortalité des enfants: relation confirmée par les résultats des enquêtes EDS à travers le monde. 
Les différences de mortalité selon le niveau d'instruction de la mère sont nettement plus importantes entre $I$ et 5 ans qu'à moins d'un an. C'est au-delà d'un an que l'enfant court le plus de risques (sevrage, arrivée d'un autre enfant ...) et c'est là que l'instruction de la mère peut jouer pleinement (Akoto et Tabutin, 1989:38). Toutefois, l'éducation de la mère "ne joue véritablement un rôle que là où sont concentrées les ressources et les infrastructures socio-économiques et sanitaires" (Akoto, 1992 : 129). L'influence de l'instruction sur la survie infantojuvénile s'exerce par l'intermédiaire de revenus élevés et d'un meilleur accès aux services de soins (Mbacké et Van de Walle, 1989 ; Gakidou et al., 2010 ; Lankoandé et al., 2016).

L'instruction du père joue également un rôle dans la baisse de mortalité infanto-juvénile. Plus le niveau d'étude du père est élevé, plus la mortalité des enfants est faible. Mais son impact est en général inférieur à celui de la mère (Akoto et Tabutin, 1989). Par ailleurs, l'instruction combinée des parents produit un effet additif sur les niveaux de mortalité des enfants de moins de 5 ans : "à tout niveau d'instruction de la mère, plus le niveau d'instruction du père augmente, plus la mortalité baisse" (Akoto et Tabutin, 1989: 40).

La littérature rapporte que la mortalité est plus faible en ville qu'à la campagne (Maîga et Bocquier, 2016). Par ailleurs, comme pour la scolarisation, les inégalités entre milieux d'habitat sont plus importantes pour les niveaux de mortalité de $I$ à 5 ans qu'à moins d'un an, ce qui confirme la plus grande sensibilité de la mortalité juvénile aux conditions de l'environnement physique, économique, social et culturel (Esso Lasme, 2013, Brinda et al., 2015 ; Lankouandé et al., 2016). La répartition inégale des ressources entre les deux secteurs d'habitat n'est pas étrangère à cette situation. En ville, les biens et les services disponibles (éducation, santé, alimentation, revenus) augmentent les probabilités de survie des enfants au détriment du milieu rural. Ces résultats ne signifient pas nécessairement que l'urbanisation en Afrique et, en particulier au Niger, va entraîner une baisse continue de la mortalité des enfants. L'augmentation de la population urbaine s'accompagne parfois d'une paupérisation inquiétante due à l'absence d'une politique urbaine et d'aménagement du territoire satisfaisante. La ville au Niger est une mosaïque de populations, d'activités, de conditions d'existence, de niveaux de vie et de traditions disparates. Le confort qu'elle offre n'est pas également partagé, l'accès aux biens et services dépend des capacités individuelles à y recourir (Maïga et Bocquier, 2016 ; Lankouandé et al., 2016)

$\mathrm{La}$ catégorie socioprofessionnelle des parents influence également la mortalité des enfants, en tant 3789 qu'indicateur de la classe sociale, du genre et du niveau de vie (Akoto et Tabutin, 1989 ; Brinda et al., 2015). Son action s'effectue à travers l'alimentation, la qualité des soins de santé accordés à l'enfant, l'accès aux infrastructures économiques et sociales. Les enfants des cadres et techniciens supérieurs meurent en général moins que ceux dont les parents travaillent dans l'agriculture (Kaldewei, 20l0).

Dans l'impossibilité de prendre en compte tous ces déterminants ci-dessus cités, dans le cadre de cette étude, notre intérêt porte sur les caractéristiques liées à l'enfant (sexe, rang de naissance, l'intervalle intergénésique avec la naissance précédente et le poids à la naissance) qui font parties de ces multiples facteurs de la mortalité des enfants identifiés depuis longtemps. En effet, il est généralement admis dans la littérature, qu'à la naissance, les filles sont plus résistantes que les garçons : avantage inné des filles pendant la période néonatale ; moindre résistance physique des garçons aux maladies respiratoires durant le premier mois de la vie (Waldron, 1987 ; WHO, 2003). Aussi, des comportements discriminatoires à l'égard des filles ou des garçons en lien avec les valeurs culturelles ou l'allocation des ressources familiales peuvent influencer leurs chances de survie (Kaldewei, 20I0).

Les enfants de rang un ont un risque de décès plus élevé que ceux de rang supérieur (deux et trois en particulier). Les raisons de cette surmortalité sont liées aux facteurs physiologique et social, l'immaturité des jeunes mères, la fragilité du corps pour porter la grossesse (Mudubu, 1996 ; Kabir et al., $201 \mathrm{l}$; Esso Lasme, 2013). Ces enfants peuvent souffrir aussi de la non-maîtrise par la mère des techniques en matière de soins et de nutrition à apporter aux nouveau-nés (Uddin et Zakir, 2008).

Pour les enfants de rang de naissance élevé, la littérature rapporte une surmortalité pour les raisons suivantes : I) les accouchements rapprochés ne permettent pas à l'organisme de la femme de récupérer toutes ses fonctions et augmentent ainsi les risques d'un accouchement précoce et d'un bébé de petit poids à la naissance (Aaby, 1992) ; 2) le rang de naissance élevé correspond généralement à une famille nombreuse, donc à un grand nombre d'enfants qui peuvent contribuer à l'appauvrissement de cette famille. D'où le fait que les nouveaux nés dans ces types de famille (famille nombreuse) sont exposés à un risque de mortalité plus élevé que ceux nés d'une famille réduite, toute chose étant égale par ailleurs. Aussi, pour une mère ayant eu une naissance de rang élevé, il y a non seulement concurrence entre ce dernier et les premiers concernant les soins, mais aussi, la mère a tendance à se fier à son expérience 
en matière de soins, en ce qui concerne le dernier né (Rakotodrabe, 1996).

L'intervalle précédent la naissance est l'une des variables liées à l'enfant à travers laquelle on peut appréhender le risque de décès des enfants (Becher, 2004 ; Kabir et al. 20II). En principe, plus cet intervalle est important, plus grande est la chance de survie de l'enfant. Les principaux mécanismes à travers lesquels de brefs intervalles précédents augmentent les risques de décès durant la première année de vie sont essentiellement les suivants: le syndrome d'épuisement maternel qui fait référence aux conséquences d'accouchements rapprochés sur l'organisme féminin qui n'est pas en mesure de récupérer toutes ses fonctions, augmentant ainsi le risque d'un accouchement précoce et d'un bébé de petit poids à la naissance (Barbieri, 1991 ; Beck, 2009) ; l'exposition aux infections : la présence d'enfants nés à brefs intervalles l'un de l'autre augmente le risque de contracter des maladies (Aaby, 1992 ; Kabir et al., 20II) ; l'effet de compétition : nés à peu de distance l'un de l'autre les enfants sont exposés à un plus haut risque de mortalité car ils se trouvent en compétition pour les ressources familiales et les soins apportés par la mère (Desgrees , 1996 ; Kabir et al., 201 I).

Le faible poids à la naissance est fortement associé à la mortalité et la morbidité infantile (Panico et al., 2015). Les nouveau-nés dont le poids est inférieur à 2500 grammes sont considérés de faible poids. Selon I'OMS et I'UNICEF(2004), les bébés nés avec un poids entre 1000-1499 g sont considérés de très faible poids ; ceux dont le poids se situe entre 500$999 \mathrm{~g}$ sont considérés d'extrêmement faible poids. Les deux principales causes d'insuffisance de poids à la naissance sont l'accouchement prématuré (avant que 37 semaines de grossesse soient terminées) et le retard de croissance intra-utérin (RCIU) ou d'une combinaison des deux (OMS et UNICEF, 2004 ; Beck, 2009 ; Panico et al., 2015). Dans les pays où la malnutrition est fréquente, environ $80 \%$ de l'insuffisance pondérale sont imputables au retard de croissance intra-utérin due en grande partie à la malnutrition maternelle (OMS, 2007). Les bébés qui naissent de petit ou très petit poids sont plus fragiles avec pour conséquence une surmortalité infantile et divers problèmes de développement et de comportement (Panico et al., 20 I5).

\section{Données et méthodes}

Aujourd'hui, il est de plus en plus admis que la notion de développement ne doit plus être uniquement appréciée par l'amélioration des indicateurs socio-économiques, mais qu'elle doit intégrer une exigence d'équité, c'est-à-dire de respect de l'égalité des chances de chaque individu associé à une absence de privations absolues, comportant un accès pour chacun aux infrastructures sociales de base et notamment aux services de santé. Ainsi, comme hypothèse centrale nous supposons que la baisse observée de la mortalité des enfants s'accompagne d'une réduction des inégalités. En conséquence, la mortalité étant fonction de l'âge, nous supposons que pour les différents groupes d'âges retenus, les différences de mortalité observées selon les conditions de naissance de l'enfant (poids à la naissance, intervalle intergénésique précédent la naissance l'enfant, le rang de naissance, le sexe) ont été atténuées avec le temps.

Les indicateurs de mortalité utilisés sont les quotients de la mortalité : néonatale ; postnéonatale ; infantile ; juvénile. Ces quotients de mortalité sont respectivement les probabilités de décéder : avant un mois exact, entre un mois exact et 12 mois exact, avant le premier anniversaire ; après le premier et avant le cinquième anniversaire. Ces quotients sont calculés pour la période de 10 ans avant l'enquête à partir d'un nombre total d'enfants nés vivants et survivants déclaré par les femmes pour chaque enquête (tableau I).

Tableau I : année de collecte, effectif des femmes enquêtées et effectifs des naissances selon le statut de survie

\begin{tabular}{|c|l|l|l|l|}
\hline & & \multicolumn{3}{|c|}{ Effectif des naissances } \\
\hline $\begin{array}{l}\text { Année de } \\
\text { collecte }\end{array}$ & $\begin{array}{l}\text { Effectif des femmes } \\
\text { enquêtées }\end{array}$ & $\begin{array}{l}\text { Nombre d'enfants } \\
\text { survivants }\end{array}$ & $\begin{array}{l}\text { Nombre d'enfants } \\
\text { décédés }\end{array}$ & $\begin{array}{l}\text { Nombre total d'enfants nés } \\
\text { vivants }\end{array}$ \\
\hline 1992 & 6332 & 17047 & 8000 & 25047 \\
\hline 1998 & 7285 & 20651 & 9133 & 29784 \\
\hline 2006 & 8924 & 27699 & 9161 & 36860 \\
\hline 2012 & 11004 & 36222 & 9345 & 45567 \\
\hline
\end{tabular}

Source rapport EDSN : 1992, 1998, 2006 et 2012.

Pour chaque variable pouvant être une source d'inégalité, une modalité sera retenue comme catégorie de référence pour déterminer les évolutions des inégalités à partir des résultats des quatre Enquêtes Démographiques et de Santé réalisés au Niger (1992,1998, 2006, 2012). 
Comme le propose Bourgeois-Pichat $(1952,1980)$ à la première année de vie nous distinguons les décès endogènes de ceux exogènes. Les décès endogènes comprennent les décès imputables à la constitution même de l'enfant, c'est-à-dire à des causes que l'enfant porte en lui-même à la naissance, qu'il les ait héritées dès la conception ou acquises au cours de la gestation ou de l'accouchement. Les décès exogènes comprennent ceux dont la cause est extérieure à l'enfant ; c'est alors le milieu où vit l'enfant qui lui apporte les facteurs de sa mort, sous forme de mauvaise alimentation, de microbes, manque d'accès aux services de santé, discrimination sociale, etc (Henripin 196I, Bourgeois-Pichat, 1980). Cette distinction tient du fait que c'est surtout pour les décès de la dernière catégorie (exogènes) que la société peut être tenue responsable. En effet, ces décès peuvent être évités assez facilement, si l'on prend certaines précautions. En outre, la médecine dispose de moyens efficaces pour combattre les maladies exogènes ; tandis qu'il n'en va pas toujours ainsi des maladies endogènes (malformations, débilité congénitale, etc.) (Henripin, 196I).

Pour avoir une évaluation des décès exogène nous nous appuyons sur la méthode biométrique développée par Jean Bourgeois-Pichat (1980) en supposant que parmi tous les décès exogènes de la première année, $20 \%$ se produisent au cours du premier mois de la vie et tous les décès des onze derniers mois de la première année sont considérés comme exogènes. Cela veut dire qu'en multipliant par I,25 les décès des onze derniers mois, on obtient une bonne évaluation de l'ensemble des décès exogènes au cours de la première année de vie.

\section{Résultats}

\section{Evolution de la mortalité des enfants au Niger}

$\mathrm{Si}$ l'on considère la mortalité exogène (infantile exogène biométrique et juvénile) on obtient des évolutions très différentes avec la mortalité endogène
(Tableau 2). Entre 1992 et 2012, la mortalité exogène passe de 104 à $4 \mathrm{I}$ et la mortalité juvénile 221 à 94 pour mille, ce qui représente une réduction de $60 \%$ et $58 \%$, respectivement. II s'agit là, de décès qui pourraient être évités dans une grande majorité, si l'on appliquait toutes les mesures d'hygiène, d'alimentation et tous les soins curatifs connus. Ces mesures et ces soins sont simples, techniquement, mais leur application dépend d'un ensemble assez complexe de facteurs sociaux qui commandent une attention plus ou moins grande accordée aux enfants et la connaissance des conditions dont ils doivent être entourés si l'on veut leur éviter d'entrer en contact avec des agents qui peuvent provoquer leur mort. Signalons, à propos de la mortalité infantile exogène, une hausse entre 1992 et 1998. Faut-il la chercher dans la qualité des données ou à une réelle recrudescence dû à une détérioration des conditions de vie ou encore à une épidémie non maitrisée?

Le quotient de mortalité endogène a baissé lui aussi. Moins, cependant, que celui de la mortalité exogène. Entre 1992 et 2012, la baisse est d'environ $22 \%$. On peut faire remarquer une relative stabilité au cours de la période 1992-2006. Pour lutter contre cette catégorie de mortalité les centres de formation sanitaire sont encore moins bien équipés même si ailleurs il est possible de lutter contre la débilité, contre les inconvénients entraînés par la prématurité de la naissance, contre certaines causes de malformation congénitale.

En somme, même si le Niger est l'un des pays où la mortalité des enfants reste encore très élevée, de façon générale, toutes les composantes de la mortalité des jeunes enfants semblent enregistrer une baisse importante. Le rythme de cette baisse est-il différent selon caractéristiques sociodémographiques de l'enfant?

Tableau 2 : Quotients de mortalité néonatale, post-néonatale, infantile et juvénile pour la période de 10 ans précédant l'enquête

\begin{tabular}{|l|c|c|c|c|c|}
\hline Quotient de mortalité & EDSN 1992 & $\begin{array}{l}\text { EDSN } \\
1998\end{array}$ & $\begin{array}{l}\text { EDSN-MICS } \\
2006\end{array}$ & EDSN-MICS 2012 & $\begin{array}{l}\% \text { de baisse } \\
\text { depuis 1992 }\end{array}$ \\
\hline Infantile endogène biométrique & 31 & 30 & 30 & 24 & 22 \\
\hline Infantile exogène biométrique & 104 & 106 & 64 & 41 & 60 \\
\hline Juvénile & 221 & 193 & 137 & 94 & 58 \\
\hline
\end{tabular}

Source : calcul de l'auteur à partir des données des EDS 1992, 1998, 2006 et 2012

Évolution des inégalités des enfants face au risque de mortalité selon le sexe de l'enfant L'examen du tableau 3 permet de constater une surmortalité masculine en ce qui concerne la mortalité infantile endogène biométrique mais également une baisse plus prononcée de la mortalité des filles ( $48 \%$ contre $6 \%$ ). En conséquence, aucune tendance à la baisse des inégalités ne semble se dégager. En effet, à égalité de chances devant la mort en 1992, une surmortalité des garçons s'observe pour le reste de la période

http://aps.journals.ac.za 
avec une variation. En 2012, les garçons semblent courir I,8 fois plus de risques de mourir que les filles si la situation observée en 2006 peut être attribuable aux variations aléatoires ou à la qualité des données.

L'examen des évolutions au niveau de la mortalité infantile exogène biométrique permet de constater une surmortalité des filles en 1998 et en 2006 alors que pour la mortalité juvénile cette surmortalité des filles est constatée en 1992 et 1998.
En somme des inégalités devant la mort selon le sexe persistent sans une amélioration notable. Bien au contraire, on assiste à une surmortalité inattendue des filles, après la période néonatale, liées plus à des causes sociales que biologiques. Aussi, durant la période néonatale les dispositions encore en cours ne semblent pas préserver les garçons de leur fragilité.

Tableau 3 : Evolution des quotients (pour 1000) et des indices de mortalité (pour 100) enfantine pour la période de 10 ans précédant l'enquête selon le sexe de l'enfant

\begin{tabular}{|c|c|c|c|c|c|}
\hline Caractéristiques & EDSN 1992 & EDSN 1998 & EDSN 2006 & EDSN 2012 & $\%$ de baisse \\
\hline Sexe & & & & & \\
\hline \multicolumn{6}{|c|}{ Mortalité infantile endogène biométrique } \\
\hline Garçon & 31 & 39 & 35 & 29 & 6 \\
\hline Fille & 31 & 21 & 26 & 16 & 48 \\
\hline \multicolumn{6}{|c|}{ Indice de mortalité (base 100, fille) } \\
\hline Garçon & 100 & 183 & 133 & 183 & \\
\hline \multicolumn{6}{|c|}{ Mortalité infantile exogène biométrique } \\
\hline Garçon & 105 & 103 & 63 & 44 & 58 \\
\hline Fille & 103 & 110 & 65 & 40 & 61 \\
\hline \multicolumn{6}{|c|}{ Indice de mortalité (base 100, fille) } \\
\hline Garçon & 102 & 93 & 96 & 109 & \\
\hline \multicolumn{6}{|l|}{ Mortalité juvenile } \\
\hline Garçon & 212 & 184 & 138 & 94 & 56 \\
\hline Fille & 232 & 202 & 135 & 95 & 59 \\
\hline \multicolumn{6}{|c|}{ Indice de mortalité (base 100, fille) } \\
\hline Garçon & 91 & 91 & 102 & 99 & \\
\hline
\end{tabular}

Source : calcul de l'auteur à partir des données des EDS 1992, 1998, 2006 et 2012

Évolution des inégalités des enfants face au risque de mortalité selon le rang de naissance de l'enfant Une baisse de la mortalité est enregistrée pour tous les rangs de naissance et à toutes les composantes de la mortalité. Cependant, si on ne note pas de différence significative selon le rang de naissance pour la mortalité juvénile, en ce qui concerne la mortalité infantile endogène biométrique la baisse est au moins deux fois plus élevée pour les naissances de premier rang comparativement aux autres rangs de naissance alors que pour la mortalité infantile exogène biométrique c'est une situation inverse qui s'observe (Tableau 4).

Toutefois, cette baisse constante de la mortalité infantile (endogène et exogène), au premier rang n'est pas suffisante pour une réduction substantielle des inégalités avec les rangs de naissance pour lesquels le niveau de mortalité est plus faible. En effet, prenant comme base 100 la mortalité des enfants de rang compris entre 4 et 6 , pour qui on observe les niveaux de mortalité les plus faibles, les http://aps.journals.ac.za inégalités face à la mortalité infantile endogène biométrique des enfants de premier rang persistent encore. Le risque de décéder de ces enfants étant de 2,9 fois plus élevé en 1992 se situe encore à 2,3 en 2012 en passant par I,8 en 2006. Moins de disparité sont observées en ce qui concerne la mortalité infantile exogène biométrique et la mortalité juvénile. Si les inégalités ne semblent pas s'aggraver il est également difficile de conclure une nette amélioration de la situation (Tableau 4).

En ce qui concerne les naissances de rang élevé, $7^{\text {ième }}$ enfants et plus, on peut noter également une baisse constante de la mortalité infantile exogène et de la mortalité juvénile. Cette baisse, au même niveau que celui des autres rangs de naissance a permis d'observer une relative stabilité des écarts observés, au cours de la période, avec les enfants de rang 4 et 6 , catégorie de référence dans le cadre de cette étude. Notons d'ailleurs que ces écarts ne semblent pas indiquer des inégalités devant la mort statistiquement significatives. 
Au Niger, tout porte à croire que le rang en tant que tel n'est pas source d'inégalité devant la mort grâce à une forme de solidarité de la famille élargie encore persistante qui permet d'apporter une aide à une mère lorsqu'elle est partagée entre deux (ou plus) enfants de bas âge ou encore au moment de la naissance de son premier.

Tableau 4 : Evolution des quotients (pour 1000) et des indices de mortalité (pour 100) enfantine, pour la période de 10 ans précédant l'enquête, selon le rang de naissance de l'enfant

\begin{tabular}{|c|c|c|c|c|c|}
\hline Caractéristiques & EDSN 1992 & EDSN 1998 & EDSN 2006 & EDSN 2012 & $\%$ de baisse \\
\hline \multicolumn{6}{|l|}{ Rang de naissance } \\
\hline \multicolumn{6}{|c|}{ Mortalité infantile endogène biométrique } \\
\hline 1 & 56 & 57 & 47 & 39 & 67 \\
\hline $2-3$ & 26 & 25 & 22 & 19 & 29 \\
\hline $4-6$ & 20 & 21 & 26 & 17 & 14 \\
\hline 7 ou plus & 37 & 26 & 32 & 28 & 24 \\
\hline \multicolumn{6}{|c|}{ indice de mortalité (base 100, 4-6) } \\
\hline 1 & 288 & 275 & 183 & 231 & \\
\hline $2-3$ & 133 & 122 & 86 & 110 & \\
\hline 7 ou plus & 188 & 127 & 124 & 166 & \\
\hline \multicolumn{6}{|c|}{ Mortalité infantile exogène biométrique } \\
\hline 1 & 109 & 105 & 69 & 46 & 31 \\
\hline $2-3$ & 95 & 109 & 59 & 38 & 57 \\
\hline $4-6$ & 108 & 101 & 66 & 41 & 61 \\
\hline 7 ou plus & 106 & 114 & 65 & 46 & 62 \\
\hline \multicolumn{6}{|c|}{ indice de mortalité (base 100, 4-6) } \\
\hline 1 & 101 & 104 & 104 & 112 & \\
\hline $2-3$ & 88 & 107 & 89 & 91 & \\
\hline 7 ou plus & 99 & 112 & 98 & 112 & \\
\hline \multicolumn{6}{|l|}{ Mortalité juvénile } \\
\hline 1 & 207 & 191 & 143 & 94 & 55 \\
\hline $2-3$ & 225 & 189 & 137 & 93 & 59 \\
\hline $4-6$ & 218 & 199 & 129 & 93 & 57 \\
\hline 7 ou plus & 237 & 190 & 143 & 99 & 58 \\
\hline \multicolumn{6}{|c|}{ indice de mortalité (base 100, 4-6) } \\
\hline 1 & 95 & 96 & 111 & 101 & \\
\hline $2-3$ & 103 & 95 & 106 & 100 & \\
\hline 7 ou plus & 109 & 95 & 111 & 106 & \\
\hline
\end{tabular}

Source : calcul de l'auteur à partir des données des EDS 1992, 1998, 2006 et 2012

Évolution des inégalités des enfants face au risque de mortalité selon l'intervalle avec la naissance précédente.

Durant la période couverte par cette étude, on note une mortalité différentielle selon la longueur de l'intervalle précédent la naissance de l'enfant. En comparant les niveaux de mortalité de 1992 et ceux de 2012 on constate que la baisse est plus prononcée pour la mortalité infantile endogène pour les enfants nés après un intervalle de moins deux ans mais, on observe l'inverse pour la mortalité infantile exogène et la mortalité juvénile (Tableau 5).

En prenant comme base 100 le niveau de mortalité des enfants nés après un intervalle de 2-3 ans on peut constater que les inégalités face à la mortalité infantile endogène biométrique des enfants nés après un intervalle de moins de deux ans n'ont pas enregistré un changement sensible. Ces enfants cours encore environ 2 fois plus de risque de mourir que ceux dont l'intervalle précédent est compris entre 2 et 3 ans 
Tableau 5 : Evolution des quotients (pour 1000) et des indices de mortalité (pour 100) enfantine, pour la période de 10 ans précédant l'enquête, selon l'intervalle intergénésique avec la naissance précédente

\begin{tabular}{|c|c|c|c|c|c|}
\hline Caractéristiques & EDSN 1992 & EDSN 1998 & EDSN-MICS 2006 & EDSN-MICS 2012 & $\%$ de baisse depuis 1992 \\
\hline \multicolumn{6}{|c|}{ Intervalle avec la naissance précédente } \\
\hline \multicolumn{6}{|c|}{ Mortalité infantile endogène biométrique } \\
\hline Moins de 2 ans & 44 & 40 & 45 & 30 & 56 \\
\hline $2-3$ ans & 22 & 18 & 20 & 16 & 25 \\
\hline 4 ans ou plus & 9 & 13 & 11 & 12 & -28 \\
\hline \multicolumn{6}{|c|}{ Indice de mortalité (base 100, 2-3 ans) } \\
\hline Moins de 2 ans & 202 & 218 & 228 & 186 & \\
\hline 4 ans ou plus & 41 & 70 & 54 & 71 & \\
\hline \multicolumn{6}{|c|}{ Mortalité infantile exogène biométrique } \\
\hline Moins de 2 ans & 115 & 136 & 85 & 59 & 49 \\
\hline $2-3$ ans & 96 & 99 & 56 & 34 & 65 \\
\hline 4 ans ou plus & 65 & 66 & 36 & 28 & 58 \\
\hline \multicolumn{6}{|c|}{ Indice de mortalité (base 100, 2-3 ans) } \\
\hline Moins de 2 ans & 119 & 138 & $|5|$ & 174 & \\
\hline 4 ans ou plus & 68 & 67 & 64 & 81 & \\
\hline \multicolumn{6}{|l|}{ Mortalité juvenile } \\
\hline Moins de 2 ans & 223 & 239 & 177 & 125 & 44 \\
\hline $2-3$ ans & 237 & 187 & 123 & 84 & 65 \\
\hline 4 ans ou plus & 157 & 89 & 73 & 49 & 69 \\
\hline \multicolumn{6}{|c|}{ Indice de mortalité (base 100, 2-3 ans) } \\
\hline Moins de 2 ans & 94 & 128 & 144 & 149 & \\
\hline 4 ans ou plus & 66 & 48 & 59 & 58 & \\
\hline
\end{tabular}

Source : calcul de l'auteur à partir des données des EDS 1992, 1998, 2006 et 2012

S'agissant de la mortalité infantile exogène biométrique les inégalités se creusent davantage entre les enfants nés après un intervalle de moins de deux ans et ceux nés après un intervalle de 2-3 ans. Un enfant né en 1992 après un intervalle de moins deux ans, cours I,2 fois plus de risque de décéder que celui né après un intervalle de 2-3 ans. Ce risque passe à I,7 en 20I2. Du côté de la mortalité juvénile, à partir de 1998 on observe une surmortalité des enfants nés après un intervalle de moins de 2 ans. Cette surmortalité semble s'aggraver entre 1998 et 2006 puis entre 2006 et 2012 . L'indice de mortalité passe de 0,9 à I,5(Tableau 5).

Par ailleurs, si les enfants nés après un intervalle de 4 ans et plus conservent toujours le plus faible niveau de mortalité durant la période, on peut remarquer que pour la mortalité infantile endogène, les inégalités entre les enfants nés après un intervalle de 2-3 ans et ceux de 4 ans ou plus semblent connaitre une réduction. L'indice de mortalité passe de 0,4 en 1992 à 0,7 en 2012 en passant par 0,5 en 2006 (Tableau 5). De même, la surmortalité infantile exogène des enfants nés après un intervalle 2-3 ans par rapport à ceux nés après un intervalle de 4 ans ou plus est relativement stable. L'indice de mortalité passe de 0,68 à 0,7 au cours de la période. Cependant, la surmortalité juvénile des enfants nés après un intervalle de 2-3 ans par rapport à ceux nés après un intervalle de 4 ans ou plus ne semble pas connaitre une amélioration. Bien au contraire, on note une légère aggravation des inégalités. L'indice de mortalité passe de 0,66 à 0,58 montrant ainsi l'aggravation de l'écart entre les deux groupes d'enfants (Tableau 5).

Évolution des inégalités des enfants face au risque de mortalité selon le poids à la naissance

Du tableau 6, il ressort que, à l'exception du pic observé en 2006, la mortalité infantile endogène des bébés de petit/très petit poids est en baisse alors que celle des bébés de poids moyen/gros reste constante durant la période. Pour la mortalité infantile exogène, on note une baisse régulière, indépendamment du poids à la naissance. Cependant la baisse semble être plus forte pour les enfants de poids moyen/gros ( $52 \%$ contre $31 \%)$. Cela se traduit par une tendance à la hausse des inégalités devant la mort en fonction du poids de l'enfant à la naissance. Cependant, une mauvaise appréciation du poids à la naissance, par des parents souvent analphabètes, en 
l'absence de carnet de santé de l'enfant, nous amène

à prendre ces résultats avec beaucoup de réserve.

Tableau 6 : Evolution des quotients (pour 1000) et des indices de mortalité (pour 100) infantile, pour la période de 10 ans précédant l'enquête, selon le poids à la naissance

\begin{tabular}{|c|c|c|c|c|c|}
\hline Caractéristiques & EDSNI 992 & EDSN 1998 & EDSN-MICS 2006 & EDSN-MICS 2012 & $\begin{array}{l}\% \text { de baisse depuis } \\
1992\end{array}$ \\
\hline \multicolumn{6}{|c|}{ Mortalité infantile endogène biométrique } \\
\hline \multicolumn{6}{|l|}{ Poids à la naissance } \\
\hline Très petit ou petit & 31 & 22 & 44 & 10 & 69 \\
\hline Moyen ou gros & 15 & 22 & 15 & 15 & 0 \\
\hline \multicolumn{6}{|c|}{ Indice de mortalité (base 100, moyen ou gros) } \\
\hline Très petit ou petit & 203 & 100 & 293 & 64 & \\
\hline \multicolumn{6}{|c|}{ Mortalité infantile exogène biométrique } \\
\hline Très petit ou petit & 110 & 79 & 65 & 53 & 31 \\
\hline Moyen ou gros & 94 & 79 & 55 & 31 & 52 \\
\hline \multicolumn{6}{|c|}{ Indice de mortalité (base 100, moyen ou gros) } \\
\hline Très petit ou petit & 117 & 100 & 118 & 168 & \\
\hline
\end{tabular}

Source : calcul de l'auteur à partir des données des EDS 1992, 1998, 2006 et 2012

\section{Discussions}

Nos résultats ont permis de mettre en évidence la persistance d'une surmortalité féminine pour la mortalité exogène. II peut s'agir d'un comportement discriminatoire à l'égard des filles ou une plus grande vulnérabilité des filles à une ou des maladies qui sont des causes prédominantes de décès à cet âge. En effet, les causes exogènes de mortalité deviennent prédominantes après la période néonatale. Au Niger ces causes sont surtout les maladies infectieuses et parasitaires, notamment la rougeole, la diarrhée, les maladies respiratoires et le paludisme.

Des études menées dans le sahel attestent que la rougeole est lus fatale aux filles qu'aux garçons (Cantrelle et al. 1986 ; Fragues et Nassour, 1988 ; Mbaké et LeGrand, 1992). Aussi, une discrimination dans l'alimentation et/ou les soins sanitaires reçus, ou bien des comportements discriminatoires exposent de manière différente les deux sexes au risque de décéder. Cette discrimination serait liée à une préférence manifeste à l'égard des garçons ; qui auraient une plus grande utilité économique, une assurance sociale que leurs sœurs (Das Gupta, 1987). Par ailleurs, selon l'UNFPA (2006), le nombre élevé d'enfants dans le ménage ne joue pas en général en faveur des filles: dans les familles nombreuses, les enfants sont ordinairement moins bien nourris et éduqués que dans les familles peu nombreuses et, ce sont surtout les filles qui en font les frais.

La baisse constante de la mortalité infantile (endogène et exogène), au premier rang, pourrait s'expliquer par le fait qu'hier comme aujourd'hui la naissance du premier enfant met en contribution, dès les premiers jours, un membre de la famille de la

3795 jeune mère pour pallier à son inexpérience dans la prise en charge du nouveau-né, aussi bien en campagne comme en ville. Elle s'explique également par l'amélioration des consultations prénatales et les sensibilisations à travers les médias.

Les inégalités persistantes devant la mort des enfants nés après un intervalle précédent de moins de 24 mois fait de l'établissement d'un programme de planification familiale une stratégie politique indispensable. L'amélioration de la prévision des besoins contraceptifs et de la logistique est impérative, les ruptures de stocks de moyens modernes de contraception sont fréquents dans de nombreuses formations sanitaires du pays. La sécurisation des produits contraceptifs est plus qu'une nécessité.

Même si les données sur le poids à la naissance sont de moindre qualité, un fait semble être certain, au Niger, le faible poids à la naissance est un des déterminants de la mortalité infantile et les inégalités devant la mort selon le poids à la naissance semblent connaitre une sensible augmentation dans le temps. S'il est difficile de lutter contre les facteurs endogènes, la lutte contre la pauvreté et l'amélioration du statut socioéconomique de la mère relève de la responsabilité sociétale et devrait faire partie des priorités des acteurs de développement pour lutter contre les inégalités devant la mort liées au faible poids à la naissance.

\section{Conclusion}

Rappelons que l'objectif de cette étude est d'examiner, à partir des données des quatre EDS, les évolutions des inégalités devant la mort observées 
selon les caractéristiques liées à l'enfant. II s'agit du sexe, du rang et du poids à la naissance de l'enfant mais aussi de l'intervalle intergénésique précédent la naissance. Pour chacun de ces déterminants il s'agit de mettre en évidence l'atténuation et/ou l'augmentation des inégalités des enfants devant la mort. A l'exception du rang de naissance, les résultats permettent de conclure que pour l'ensemble des variables retenues aucune atténuation des inégalités devant la mort ne semble s'observer.

Les inégalités devant la mort selon le sexe de l'enfant persistent sans une amélioration notable. Bien au contraire, on assiste à une surmortalité inattendue des filles, après la période néonatale, liées plus à des causes sociales et/ou biologiques. En outre, pendant la période néonatale les dispositions encore en cours ne semblent pas être suffisantes pour réduire la surmortalité des garçons par rapport aux filles. En prenant comme base 100 le niveau de mortalité des enfants nés après un intervalle de 2-3 ans on peut constater que les inégalités face à la mortalité infantile endogène restent invariantes par rapport à ceux nés après un intervalle de moins de deux ans et, pour la mortalité infantile exogène et juvénile on note une augmentation des inégalités dans le temps. Par ailleurs, on peut remarquer une tendance à la hausse des inégalités devant la mort en fonction du poids de l'enfant à la naissance.

Les causes sociales d'une surmortalité féminine pendant la période juvénile renvoient plus à une discrimination dans l'alimentation et/ou les soins sanitaires donnés à la fille. Les garçons également ne sont pas à l'abri d'un tel comportement. S'il peut être admissible qu'un couple adopte un certain nombre de comportements avant et pendant la grossesse pour obtenir un sexe déterminé de l'enfant, il est moins acceptable qu'il se comporte différemment face à son enfant parce qu'il ne porte pas le sexe qu'il désir. II est à explorer pour savoir si cette préférence du sexe n'est pas en lien avec la forte fécondité que le Niger connait aujourd'hui. Un effet, un couple pourrait obtenir le nombre d'enfants désiré et arrêter de faire recours à une méthode contraceptive parce qu'il est à la recherche d'un ou des enfants d'un sexe donné.

Les inégalités aggravantes des enfants devant la mort selon l'intervalle intergénisique en appel à l'espacement des naissances. II revient également de chercher à répertorier et d'éliminer tous les principaux obstacles qui s'opposent encore à l'utilisation des services de planification familiale. Le gouvernement du Niger, les organismes et les donateurs internationaux et tous ceux et celles voués à extraire les peuples de la pauvreté et à améliorer la santé des femmes et des enfants doivent impérativement s'unir en un effort vigoureux de sélection des meilleures pratiques des programmes de planification familiale d'antan et de mise en œuvre de ces pratiques au service du lancement d'une planification familiale volontaire à grande échelle.

Si le régime alimentaire maternel avant et durant la grossesse a une influence majeure sur le poids de l'enfant à la naissance on ne doit pas occulter le syndrome d'épuisement maternel qui fait référence aux conséquences d'accouchements rapprochés sur l'organisme féminin qui n'est pas en mesure de récupérer toutes ses fonctions, augmentant ainsi le risque d'un accouchement précoce et d'un bébé de petit poids à la naissance. Par ailleurs, il semble qu'à l'âge adulte ceux qui sont nés de petit poids risquent plus de faire de l'hypertension, d'avoir du diabète et des maladies cardio-vasculaires surtout s'ils ont eu un retard de croissance intra-utérin. II est donc important de comprendre ce qui entraîne une naissance de petit poids et de tenter de prévenir ce problème.

Par ailleurs, l'approche descriptive utilisée dans le cadre de cette étude ne permet pas de comprendre véritablement les causes sous-jacentes de l'évolution des inégalités observées. Après cette mise en évidence des différentes tendances, dans une seconde étape, nous nous proposons de chercher à comprendre les causes sous-jacentes en faisant recours à des techniques plus appropriées.

\section{Bibliographies}

AABY P., 1992, "Overcrowding and intensive exposure: major determinants of variations in measles mortality in Africa", in E. Van de Walle, G.Pison and M. Sala Diakanda (Eds), Mortality and Society in Sub Saharan Africa, Clarendon Press, Oxford.

AKOTO E.M., 1992, Mortalité et morbidité des enfants, in AKOTO E., GUINGNIDO J.G., et TABUTIN D., Ceds, L'état et le devenir de la population du Bénin, Porto-Novo, CNPMS, pp. I55- 198.

AKOTO E.M., 1993, Déterminants socioculturels de la mortalité des enfants en Afrique Noire, Hypothèses et recherche d'explication, Louvain la Neuve, Académia.

AKOTO E.M., 1989, Déterminants socioculturels de la mortalité des enfants en Afrique Noire. Hypothèses et recherche d'explication au Cameroun, au Kenya et au Sénégal), Thèse de Doctorat, Institut de Démographie, Université Catholique de Louvain.

AKOTO E.M. et TABUTIN D., 1989, "Les inégalités socioéconomiques et culturelles devant la mort." In PISON G., VAN de WALLE E.et SALADIAKANDA (eds.) Mortalité et Société en Afrique, Paris, INED, Travaux et Documents, Cahier no 124, pp. 35-63. 
ATTAMA S., MISCKA S. KOURGUENI A. I., KOCHE H., BARRERE B., 1998, Enquête Démographique et de Santé du Niger 1998. Calverton, Maryland, USA, CARE International/Niger et Macro International Inc.

BARBIERI M., |99|, "Les déterminants de la mortalité des enfants dans le tiers monde», les dossiers du CEPED, $\mathrm{N}^{\circ}$ I8, Paris, 40p.

BECK L., 2009, la santé des nouveau-nés au Rwanda, évolution des facteurs associés aux tendances de la mortalité néonatale, S.F.S.P. "Santé Publique », Vol. 21 pp. 159 à 172

BECHER ․․, OLAF M., ALBRECHT J., ADJIMA G., KYNAST-WOLF $\underline{G}$. , AND KOUYATÉ $\underline{B}$, 2004, «Risk factors of infant and child mortality in rural Burkina Fasom, Bulletin of the World Health Organization, 2004, 82, pp.:265-273.

BOURGEOIS-PICHAT J., 1980, « Les causes de la mortalité infantile dans les pays développés au cours des toutes dernières années ", dans mortalité dans le monde et dans l'histoire, ORDINA, PP. 159-197.

BRINDA E. M., RAJKUMAR A. P , AND ENEMARK U., 2015, " Association between gender inequality index and child mortality rates: a cross-national study of 138 countries", $\underline{B M C}$ Public Health, vol. I5, pp. I449-I453.

CANTRELLE P. et LOCOH T., 1990, «Facteurs culturels et sociaux de la santé en Afrique de I'Ouest ", Les Dossiers du CPED, $\mathrm{N}^{\circ} 10$, Paris, CEPED.

CANTRELLE P., DIOP I., GARENNE M., GUEYE MET SADIO A (1986) "The profile of mortality and its determinants in Senegal" 1960-t980', dans Determinants of mortality change and differentials in developing countries, United Nations, ST/ESA/SER.A/94),PP-8 6-II6.

CHESNAIS J.C., 1986, La transition démographique : étapes, formes, implications économiques, Travaux et Documents, Cahiers $n^{\circ} 113$, INED, Paris.

CROZE M., 1963, "Mortalité en France suivant le milieu social ", acte du congrès de I'UIESP, OTTAWA, PP.263-285.

DAS GUPTA M, 1987, "selective discrimination against female children in rural Punjab"

India, Population and development Review. vol. 3, I, pp. 77-I00.

DEMENY P., 1965, "Investment Allocation and Population Growth", Demography, vol. 2: 203232.

DESGREES D. L., 1996, "Sauver les vies : le rôle des vaccinations ", les études du CEPED N ${ }^{\circ} 12$.
DINH Q. C., 1998, "Les inégalités sociales de la mortalité infantile s'estompent ", Economie et Statistique, vol. 314,I pp. 89-106.

ESSO LASME J. C. E., 2013, "Les déterminants de la mortalité des enfants de moins cinq ans en Côte d'Ivoire "European Scientific Journal, vol.9, No.2 pp. |857-788|.

FRAGUES P. et NASSOUR O. 1988, Douze ans de mortalité au Sahel: niveau, tendance, saison et causes, de mortalité à Bamako 1974-1985- INED, Paris, Travaux et Documents 123, $198 \mathrm{p}$.

GAKIDOU E., COWLINNG K., LOZONA R. ET MURRAY C. J., 2010, «Increased educational attainment and its effect on child mortality in 175 countries between 1970and 2009 : a systematic analysis», Lancet, 376(9745), pp. 959-974.

HAMADOU DAOUDA Y., 2012, Déterminants de la mortalité infantile et infanto-juvénile et la pauvreté au Niger, Revue d'Economie Théorique et Appliquée, Volume 2- Numéro I, pp 23-47.

HENRIPIN J.196I. "L'inégalité sociale devant la mort: la mortinatalité et la mortalité infantile à Montréal "Recherches sociographiques, vol. 2, no I, janvier-mars I96I, pp. 3-34.

INSTITUT NATIONAL DE LA STATISTIQUE (INS) et ICF International Inc., 2013, Enquête Démographique et de Santé et à Indicateurs Multiples du Niger 20/2. Calverton, Maryland, USA : INS et ICF International.

INSTITUT NATIONAL DE LA STATISTIQUE (INS) et Macro International Inc., 2007,. Enquête Démographique et de Santé et à Indicateurs Multiples du Niger 2006. Calverton, Maryland, USA : INS et Macro International Inc.

INSTITUT NATIONAL DE LA STATISTIQUE (INS) et Macro International Inc., 1993, Enquête Démographique et de Santé du Niger 1992. Calverton, Maryland, USA : INS et Macro International Inc.

KABIR M.A., AL-AMIN A. Q., GAZI M. A. AND MATIN M.A., 20II. "Early Childhood Mortality and Affecting Factors in Developing Countries: An Experience from Bangladesh». International Journal of Pharmacology, 7, PP. 790-796.

KALDEWE C., 2010, Determinants of Infant and Under-Five Mortality - The Case of Jordan

Technical note, Economic Affairs Officer at the Development Policy and Analysis Division of the United Nations, Department of Economic and Social Affairs (DPAD/UN-DESA).

LANKOANDE B., SOURA A. , MILLOGO R., COMPAORE Y., ROSSIER C., 2016, " surmortalité des enfants dans les quartiers informels de Ouagadougou : effet de composition 
ou effet de contexte ", African population studies, vol 30, $n^{\circ}$ I, pp.-2192-2202.

MAÏGA A. ET BOCQUIER Ph. 2016, "Dynamiques urbaines et santé des enfants en Afrique subsaharienne ", African population studies, vol 30, n I, pp.-22।3-2226.

MBACKE C. et VAN de WALLE, 1989, "Les facteurs socioéconomiques et I 'influence de la fréquentation des services de santé ", in PISON G., VAN de WALLE LA-DIAKANDA, Mortalité et Société en Afrique, Paris, INED, Travaux et Documents, Cahier no 124, chap. 3, pp. 67-84.

MBACKE C. S. M. et LEGRAND T. K., 1992, " Différences de mortalité selon le sexe et utilisation de services de santé au Mali ". Cahiers Québéquois de démographie, vol.21, n० 1992. pp99-II9.

McKEOWN T., 1976, the modern rise of population, Edward Arnold, Londres.

MOSLEY W.H., 1985, " Les soins de santé primaire peuvent-ils réduire la mortalité infantile ? Bilan critique de quelques programmes africains et asiatiques ", in VALLIN J. et LOPEZ A. (éds), La lutte contre la mort : influence des politiques sociales et des politiques de santé sur l'évolution de la mortalité, Travaux et Documents, Cahier $n^{\circ}$ 108, Paris, INED-PUF : I05-136.

MUDUBU L., 1996, Mortalité infantile et juvénile au Togo : contribution des facteurs socioéconomiques et culturels. Les cahiers de l'IFORD $\mathrm{n}^{\circ} \mathrm{Il}$. Yaoundé.

RAKOTONDRABE F. P. ,1996, "Les facteurs de la mortalité des enfants à Madagascar ». Les cahiers de l'IFORD n ${ }^{\circ} 12$, Yaoundé.

PANICO L., TO M. et THEVENON O., 20I5, " La fréquence des naissances de petit poids : quelle influence a le niveau d'instruction des mères ? ", Population \& Sociétés, numéro 523.

RUTSTEIN S. O., 2000, «Infant and child mortality in developing countries during the 1990s", Bulletin of the World Health Organization, 2000, 78 (I0), pp. 1256-1270.

RAZZEL P.E., 1965, "Edward Jenner : the History of a Medical Myth”, Medical History, vol. 10, $\mathrm{n}^{\circ} 3$, pp. 216-229.

SARTORIUS B. K.D AND SARTORIUS K. , 20I4, "Global infant mortality trends and attributable determinants - an ecological study using data from 192 countries for the period 1990-2011", Population Health Metrics, pp. I2:29.
STOLNITZ G.H., 1965, " Recent mortality trends in Latin America, Asia and Africa ", Population Studies, vol. 19 : I17-138.

TABUTIN D., 1995, " Transitions et théories de mortalité ", in GERARD H. et PICHE V. (eds.), La sociologie des populations, Collection Universités francophones, PUM/AUPELF-UREF, Montréal : 257-288.

UDDIN J., AND ZAKIR H., 2008, Predictors of Infant Mortality in a Developing Country. Asian Journal of Epidemiology Vol I(I), pp. I-I6.

UNITED NATIONS CHILDREN'S FUND AND WORLD HEALTH ORGANIZATION, 2004, Low Birthweight: Country, regional and global estimates. UNICEF, New York, 27p.

VALLIN J., 1989, " Théorie(s) de la baisse de la mortalité et situation africaine ", in PISON G., van de WALLE E. et SALA-DIAKANDA M. (éds.), Mortalité et société en Afrique, Travaux et Documents, Cahier $n^{\circ}$ 124, INED-UIESP-IFORDMNHN, PUF, Paris : 399-43I.

WALDRON I., 1987, "Profils et causes de la surmortalité féminine chez les enfants dans les pays en développement ", Rapport Trimestriel de Statistiques Sanitaires Mondiales, vol. 40, 3, OMS, Genève, pp. 194-210.

WHO and All Party Parliamentary Group on Population, Development and Reproductive Health (APPG PD\&RH), 2007, -Return of the Population Growth Factor: It's Impact upon the Millennium Development Goals, London: APPG PD\&RH, 2007, $32 \mathrm{p}$.

WHO, 2003, World Health Report 2003: Shaping the future. Geneva.

WHO, 1988 , Guidelines for implementing the Bamako Initiative Regional Committee for Africa, 38th session. Brazzaville.

WHO 1978, Declaration of Alma-Ata, International conference on Primary Health Care. Alma-Ata, USSR.

YOU D., HUG L., ELDEMY S., IDELE P.HOGAN D., MATHERS C., GERLAND P., NEW J.R. ET ALKEMA L., 2015, "Global, regional, and national levels and trends in under-5 mortality between 1990 and 2015, with scenario-based projections to 2030: a systematic analysis by the UN Interagency Group for Child Mortality Estimation", Lancet, 386 (I00 I0): 2275-2286. 